\title{
Tromboembolismo pulmonar bilateral como presentación de un síndrome antifosfolipídico: un reporte de caso
}

\section{Bilateral pulmonary thromboembolism as the presenting manifestation of the antiphospholipidic syndrome: report of a case}

Daniel Vásquez-Álvarez ${ }^{1,2, a}$, Johan Azañero-Haro ${ }^{3}$, Isabel Suni Jiménez-Casaverde ${ }^{2,4, a}$, Alonso SotoTarazona $3,4,5$

1 Facultad de Medicina. Universidad Nacional Mayor de San Marcos. Lima, Perú.

2 Departamento de Neumología. Hospital Nacional Hipólito Unanue. Lima, Perú.

3 Departamento de Medicina Interna. Hospital Nacional Hipólito Unanue. Lima, Perú.

4 Facultad de Medicina. Universidad Ricardo Palma. Lima, Perú.

5 Instituto de Investigación en Ciencias Biomédicas (INICIB). Lima, Perú.

a Médico Residente de Neumología.

Correspondencia Daniel Vásquez Alvarez dvasal@hotmail.com

Recibido: 16/08/2018

Arbitrado por pares

Aprobado: 24/10/2018

Citar como: Vásquez-Álvarez D, Azañero-Haro J, Jiménez-Casaverde IS, Soto-Tarazona A. Demanda en servicios pediátricos de un hospital del Callao, Perú. Acta Med Peru. 2018;35(4):239-43

\section{RESUMEN}

Aunque la tromboembolia pulmonar es frecuente en aquellos pacientes con síndrome antifosfolipidico (SAF), la presentación bilateral no es usual, así como la presentación en adultos mayores. Se presenta el caso de un paciente varón, adulto mayor de 72 años con antecedente de purpura trombocitopénica inmune quien fue hospitalizado con el diagnóstico de tromboembolia pulmonar bilateral. Diecisiete días después del ingreso se realizó el diagnostico de SAF primario y tuvo una buena evolución clínica. Se debe considerar SAF primario en aquellos pacientes con eventos vasculares trombóticos en ausencia de condiciones predisponentes.

Palabras clave: Embolia pulmonar; Síndrome antifosfolípido; Adulto Mayor; Púrpura trombocitopénica (fuente: DeCS BIREME).

\section{ABSTRACT}

Although pulmonary embolism is frequent in the antiphospholipid syndrome (APS), bilateral involvement is not usual, as well as its occurrence in the elderly. We present the case of a 72-year old subject with a past medical history of autoimmune thrombocytopenic purpura, who was admitted because of bilateral pulmonary thromboembolism. Seventeen days after admission, a diagnosis of primary APS was made, and the patient did well. Primary APS should be considered in patients with thrombotic vascular events, in the absence of other predisposing conditions.

Keywords: Pulmonary embolism; Antiphospholipid syndrome; Purpura, thrombocytopenic (source: MeSH NLM). 


\section{INTRODUCCIÓN}

El síndrome antifosfolipídico (SAF) es una entidad clínica sistémica, que se caracteriza por presencia de anticuerpos dirigidos contra los fosfolípidos de las proteínas del plasma, ligadas a fosfolípidos aniónicos y que combina una o varias de las siguientes manifestaciones clínicas: a) trombosis venosas repetidas; b) accidentes arteriales que afectan al sistema nervioso central; c) muertes fetales recidivantes; y d) trombocitopenia. Este síndrome ocurre principalmente en mujeres jóvenes de edad fértil y solo $12 \%$ de los pacientes superan los 50 años ${ }^{[1]}$.

EL SAF puede ser primario, cuando no está asociado a otra enfermedad o secundario cuando está asociado a una enfermedad autoinmune por ejemplo lupus eritematoso sistémico $o$ artritis reumatoide. La frecuencia del SAF primario en la población general es baja, pero su cifra exacta es desconocida ${ }^{[1,2]}$.

Las trombosis constituyen su principal complicación clínica y se caracterizan por su alta recurrencia; ya que pueden afectar a vasos tanto arteriales como venosos. Las más frecuentes son las que afectan al sistema venoso profundo de las extremidades inferiores, que pueden acompañarse de tromboembolia pulmonar en aproximadamente el $9 \%$ de $\operatorname{casos}^{[3,4]}$. En ocasiones, la recurrencia de episodios tromboembólicos a nivel del sistema arterial pulmonar, pueden conducir al desarrollo de una hipertensión pulmonar crónica incrementando de manera significativa el riesgo de morbi-mortalidad de los pacientes ${ }^{[2]}$.

\section{REPORTE DE CASO}

Varón de 72 años, con antecedentes de hipertensión arterial desde hace 5 años en tratamiento irregular, insuficiencia venosa periférica hace 2 años, y litiasis renal coraliforme con hidronefrosis derecha hace 1 mes. Adicionalmente, se encuentra que dos meses antes de su ingreso fue hospitalizado con diagnóstico clínico de Purpura Trombocitopénica Autoinmune en base a un recuento plaquetario de 20 000; sin encontrarse otras alteraciones en el hemograma ni esplenomegalia. Por estos hallazgos se inició tratamiento con pulsos de metilprednisolona seguidos por prednisona $60 \mathrm{mg} /$ día con normalización del recuento plaquetario, por lo que se disminuye la dosis progresivamente hasta $40 \mathrm{mg} /$ día, permaneciendo asintomático hasta el día de ingreso.

Dos horas antes de su ingreso presentó disnea a moderados esfuerzos, la cual fue progresando rápidamente hasta tornarse al reposo. La disnea se asociaba a dolor precordial de moderada intensidad, tipo opresivo, con irradiación al dorso, sin otros síntomas asociados, por lo que es admitido a emergencia del Hospital Nacional Hipólito Unanue. Al ingreso, el paciente cursó con PA: 130/70 mm Hg, FC: $110 x^{\prime}$, FR: 40 x', SatO2: 64\%. Al examen físico se encontró murmullo vesicular disminuido en los dos tercios inferiores del hemitórax derecho, sin otras alteraciones asociadas.
En su segundo día de hospitalización y ante persistencia de disnea se plantea el diagnostico presuntivo de tromboembolismo pulmonar. Se realiza un Doppler venoso el cual evidenció trombosis venosa profunda bilateral de ambos miembros inferiores, por lo que se inició tratamiento con enoxaparina $60 \mathrm{mg}$ c/12 horas e hidrocortisona $250 \mathrm{mg}$ EV C/8h además de oxigenoterapia. El paciente evoluciona sin mejoría clínica por lo que ingresa a la unidad de cuidados intermedios de medicina, donde nueve días después del ingreso, se le solicita una angio-TEM pulmonar, que muestra imágenes compatibles con Embolismo Pulmonar Bilateral (Figura 1).

El hemograma solicitado al ingreso mostró leucocitos en 13140 cel/ $\mu \mathrm{L}$ (segmentados $83 \%$, linfocitos $12 \%$, monocitos $5 \%$ ); hemoglobina: 10,5 gr/dL; plaquetas en 297000 cel x mm ; dimero D: $5,0 \mathrm{mcg} / \mathrm{ml}$; $\mathrm{Na}: 132 \mathrm{mEq} / \mathrm{ml} ; \mathrm{K}: 5 \mathrm{mEq} / \mathrm{ml}$; urea: $53 \mathrm{mg} / \mathrm{dL}$; creatinina: $2,41 \mathrm{mg} /$ $\mathrm{dL}$; glucosa: $175 \mathrm{mg} / \mathrm{dL}$; troponina: 0,315 ng/ml; CPK-MB: $23 \mathrm{U} / \mathrm{L}$ (VN<25) , TTP: 34,81 segundos, fibrinógeno: $560 \mathrm{mg} / \mathrm{ml}$ (VN $<400$ ), TP: 16,41 segundos, INR : 1,41. El electrocardiograma no mostró alteración. El ecocardiograma mostró distensión de la aurícula y ventrículo derecho compatibles con embolismo pulmonar.

Los anticuerpos antinucleares y el test de Coombs directo fueron negativos. Otros exámenes auxiliares mostraron anticuerpos anticitoplasma de neutrófilos (ANCA) C y P. Proteína S funcional: 72\%; proteína C funcional: 82\%; anticardiolipina IgM: 4,4 U/ $\mathrm{ml}(\mathrm{VN}:<2,0)$; antifosfatidilserina IgM: $2,7 \mathrm{U} / \mathrm{ml}(\mathrm{VN}:<2,0)$; antifosfatidilinositol IgM: $2,1 \mathrm{U} / \mathrm{ml}(\mathrm{VN}:<2,0)$; anti-beta 2 glicoproteina I IgM: 6,3 U/ml (VN<2,0).

Debido a los resultados encontrados en los anticuerpos antifosfolipidicos (anticardiolipina IgM, antifosfatidilserina IgM, antifosfatidilinositol IgM, y Anti-beta 2 glicoproteina I IgM); así como los diagnósticos de trombocitopenia autoinmune $y$ tromboembolismo pulmonar, se planteó el diagnóstico definitivo de síndrome antifosfolipídico primario (Figura 2). El paciente evolucionó favorablemente, con disminución progresiva de requerimiento de oxígeno, saliendo de alta con indicación de warfarina oral $5 \mathrm{mg} /$ día y prednisona $40 \mathrm{mg} /$ día.

\section{DISCUSIÓN}

La aparición de síndrome antifosfolipidico (SAF) se asocia, en la mitad de los casos, a otras enfermedades autoinmunes como el lupus eritematoso sistémico. Sin embargo, otros eventos menos frecuentes son la aparición de eventos tromboticos, cuando estos eventos, ya sean arteriales o venosos, ocurren en pacientes que no poseen factores de riesgo o cuando son recurrentes, se debe considerar el diagnóstico de SAF ${ }^{[5-7]}$.

Por ende, el diagnóstico se realiza en gran medida, por la sospecha clínica y por los hallazgos de laboratorio. Es así que para establecer el diagnóstico de SAF se utilizan los criterios de Sapporo, recientemente revisados ${ }^{[8]}$, en donde se especifica que para establecer el diagnóstico de SAF se requiere de al menos un criterio clínico y un criterio de laboratorio (Tabla 1). 


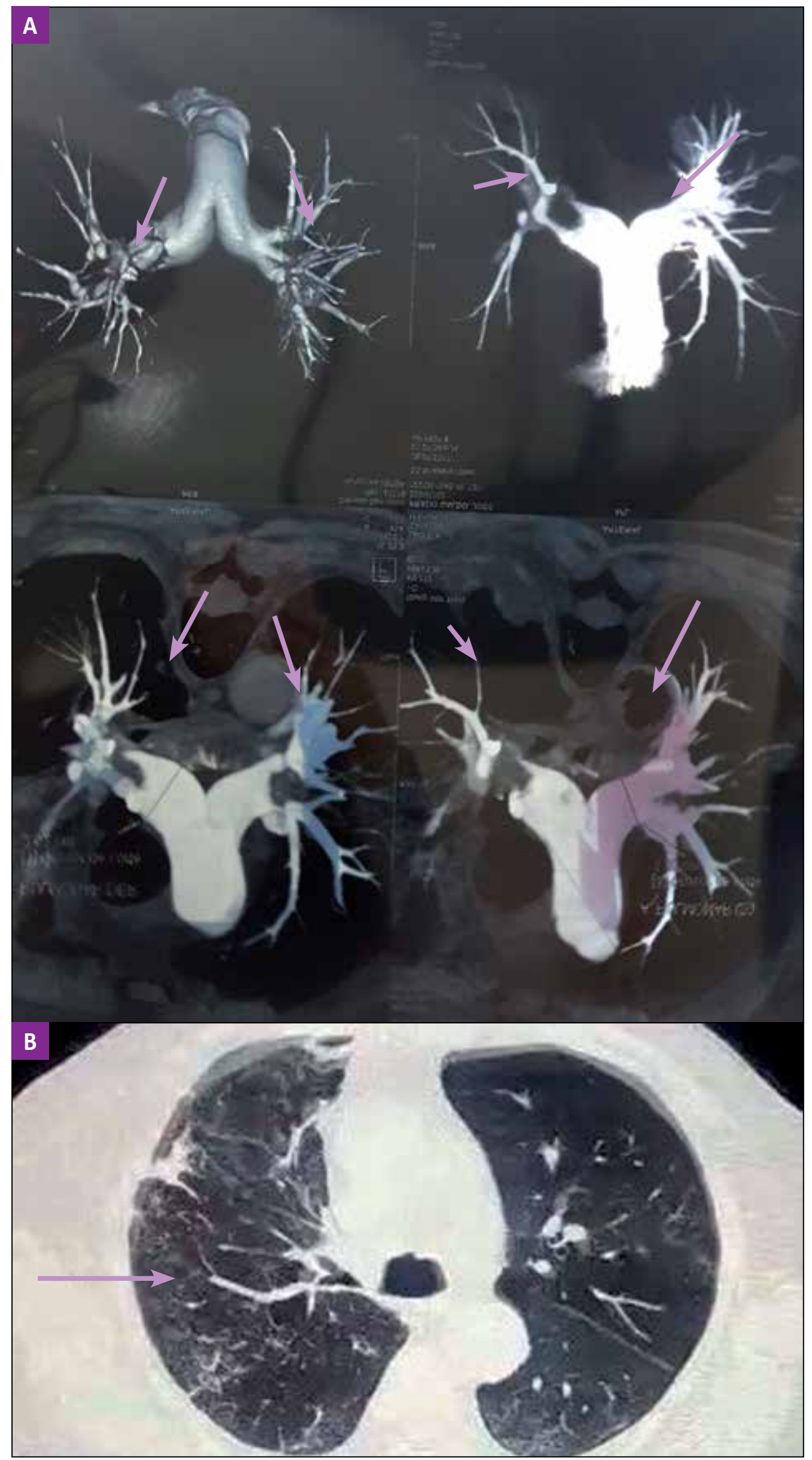

Figura 1. Exámenes de imágenes sugestivos de tromboembolismo pulmonar. A) Angio-TEM torácica mostrando (flechas) la ubicación de los trombos en las arterias pulmonares izquierda y derecha. B) Imagen de tomografía espiral multicorte (TEM) pulmonar. La flecha señala una zona compatible con infarto pulmonar a nivel de lóbulo medio derecho. 


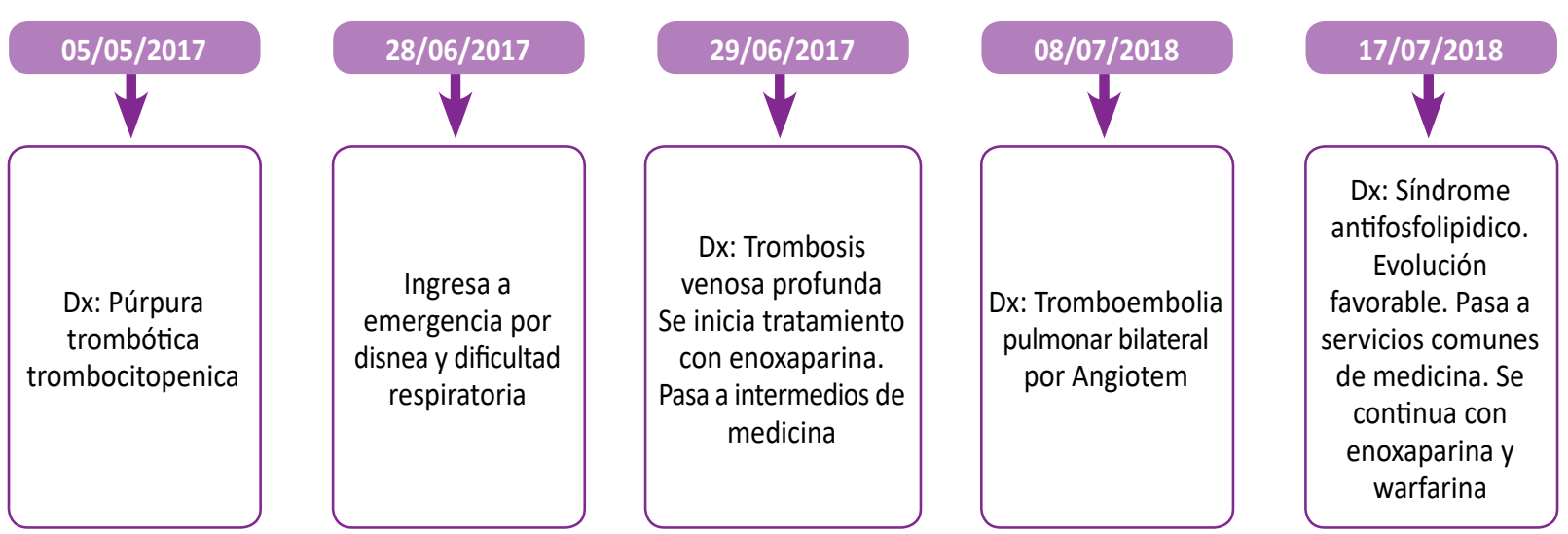

Dx: diagnóstico

Figura 2. Línea del tiempo que muestra la evolución de caso de tromboembolismo pulmonar bilateral como presentación de Síndrome antifosfolipidico.

De lo anteriormente explicado, se puede deducir que el diagnóstico de SAF se realiza por descarte, y gran parte de la guía hacia él se da por la relación entre eventos asociados 0 manifestaciones clínicas específicos asociados a el historial patológico del paciente; por ejemplo, en nuestro caso debuto con embolia pulmonar bilateral como primera manifestación de este síndrome, lo que representó una demora en establecer el diagnóstico definitivo debido a la poca frecuencia de esta manifestación en casos de SAF.

Si bien se reporta que el $40 \%$ de pacientes con SAF, presentan embolia pulmonar (EP) en el transcurso de la enfermedad ${ }^{\left[{ }^{[9]}\right.}$, solo un $9 \%$ de pacientes debutan con esta condición, y la tasa es aún más infrecuente cuando se trata de una presentación bilateral [10-12]. Los pacientes en los que se ha encontrado trombosis bilateral de las principales arterias pulmonares, en su mayoría, suelen ser pacientes con SAF primario, que desarrollaron clínica de hipertensión pulmonar (HTP) y en quienes en el estudio angiográfico demostró afectación de las arterias pulmonares, tanto de las principales como de las lobulares, en forma de obstrucción completa o parcial ${ }^{[10]}$. Se debe recordar que la tromboembolia pulmonar recurrente puede dar lugar a HTP, que puede acompañarse de compromiso valvular tricúspideo aislado, complicando aún más la posibilidad de diagnosticar un SAF ${ }^{[11,13]}$.

Adicionalmente a esto, se agrega la dificultad de poder hacer un diagnóstico definitivo de tromboembolismo pulmonar ya que éste solo se confirma mediante pruebas objetivas en un $20 \%$ de los pacientes ${ }^{[4]}$. Es así que, usualmente, el diagnóstico debe ser adaptado a la gravedad de la presentación clínica, asociado a la ausencia de antecedentes patológicos sospechosos (inmovilización prolongada, obesidad o trombofilia hereditaria o adquirida), sobre la base de si el estado del paciente es hemodinámicamente estable o inestable.

El tratamiento de la embolia pulmonar no difiere por la presencia de SAF. En el caso de que la heparina este contraindicada o existan reacciones adversas, se puede optar por anticoagulantes de acción directa (dabigatran, rivaroxaban o apixaban) mientras que la terapia trombolítica está indicada en caso de inestabilidad hemodinámica con disfunción ventricular derecha ${ }^{[14,15]}$. En nuestro paciente se optó por una terapia de warfarina $5 \mathrm{mg}$ de manera indefinida y controles periódicos del INR. La terapia a largo plazo con cumarínicos (warfarina) ha sido motivo de controversia, en cuanto al valor máximo que debe tener el INR; sin embargo, recientes estudios han mostrado que un INR 2-3 da igual beneficio que un INR mayor ${ }^{[16,17]}$.

Otro aspecto que llama la atención en este caso es la presencia de purpura trombocitopénica autoinmune (PTA) precediendo al diagnóstico de SAF; ya que, aun cuando no forma parte de los criterios diagnósticos actuales, se ha descrito la relación de diversas manifestaciones de purpura y SAF durante la evolución de estos pacientes ${ }^{[18,19]}$. La presencia de trombocitopenia representa un reto en pacientes con trombosis, quienes no tienen mayores alternativas terapéuticas que recibir anticoagulación. En el caso de nuestro paciente sin embargo el recuento de plaquetas se había normalizado previamente con la terapia esteroidea.

En este caso, se encontró que el cuadro de tromboembolismo pulmonar estuvo acompañado de trombosis venosa, que es la más frecuente manifestación de síndrome antifosfolipídico $(38,9 \%)$. Se ha descrito que hasta un $55 \%$ de pacientes con SAF cursan con trombosis venosa, particularmente de la extremidad inferior; y la mitad de ellos también presentará embolia pulmonar ${ }^{[10]}$.

Aunque el SAF suele presentarse en el contexto de enfermedades autoinmunes o neoplásicas como un fenómeno secundario, generalmente en adultos jóvenes, la presentación primaria, particularmente en el adulto mayor constituye un reto diagnóstico para el clínico. En conclusión, se debe sospechar de SAF en todo paciente que presente un evento vascular trombótico, particularmente en ausencia de condiciones predisponentes asociadas. El manejo debe ser multidisciplinario y se debe realizar una búsqueda sistemática de condiciones secundarias (particularmente enfermedades difusas del tejido conectivo) antes de calificar a un SAF como primario. 
Tabla 1. Criterios de Sapporo para diagnóstico de síndrome antifosfolipídico (*) .

\section{Criterios Clínicos:}

Trombosis vascular: uno o más episodios de trombosis arterial, venosa o de pequeños vasos en cualquier tejido u órgano.

Complicaciones del embarazo:

Una o más muertes inexplicables de fetos morfológicamente normales a las 10 semanas o más de gestación, con morfología fetal normal, o

Uno o más nacimientos prematuros de neonatos morfológicamente normales a las 34 semanas de gestación o antes, debido a eclampsia, preeclampsia severa o insuficiencia placentaria severa, o

Tres o más abortos espontáneos consecutivos inexplicables antes de la semana 10 de gestación, habiéndose excluido anormalidades maternas anatómicas u hormonales y alteraciones cromosómicas en ambos padres.

\section{Criterios de laboratorio:}

Anticoagulante lupico en plasma, en 2 o más ocasiones, con un intervalo mínimo de 12 semanas.

Anticuerpos anticardiolipina IgG y/o IgM en suero o plasma con títulos medios o altos (> $40 \mathrm{GPL}$ o MPL, o > percentil 99), en dos o más ocasiones, con un intervalo mínimo de 12 semanas, determinados por una prueba de ELISA estandarizada.

Anticuerpos anti- $\beta_{2}$ glicoproteina I lgG y/o IgM en suero o plasma con un título > percentil 99 , en más ocasiones, con un intervalo mínimo de 12 semanas, determinados por una prueba de ELISA estandarizada.

$\left({ }^{*}\right)$ Adaptado de Keeling D, et al. Guidelines on the investigation and management of antiphospholipid syndrome. Br J Haematol. 2012;157(1):47-58.

Fuente de Financiamiento: Todos los gastos en la elaboración del caso fueron cubiertos por los autores.

Conflictos de interés: No se presentó ningún conflicto de intereses entre los autores y la elaboración del presente caso.

\section{REFERENCIAS BIBLIOGRÁFICAS}

1. Cervera R. Antiphospholipid syndrome. Thromb Res. 2017;151 Suppl 1:S43-S47.

2. Bertolaccini M, Sanna G. Recent advances in understanding antiphospholipid syndrome. F1000Research. 2016;5(2908):1-9.

3. Cervera R, Piette J-C, Font J, Khamashta MA, Shoenfeld Y, Camps $\mathrm{MT}$, et al. Antiphospholipid syndrome: clinical and immunologic manifestations and patterns of disease expression in a cohort of 1,000 patients. Arthritis Rheum. 2002;46(4):1019-27.

4. Bucciarelli S, Espinosa G, Cervera R, Erkan D, Gómez-Puerta JA, Ramos-Casals $M$, et al. Mortality in the catastrophic antiphospholipid syndrome: causes of death and prognostic factors in a series of 250 patients. Arthritis Rheum. 2006;54(8):2568-76

5. Espinosa G, Cervera R, Font J. El pulmón en el síndrome antifosfolipídico. Arch Bronconeumol. 2002;38(1):27-32.

6. Shu-Hsu L, Yi-Chen W, Yi-Shan W, Shih-Chung H, Chin-Sheng Li. A Rare cause of pulmonary embolism and seizure in a young man: Antiphospholipid Syndrome. Acta Cardiol Sin. 2016;32(2):247-9.

7. Vergara CE. Trombosis biventriculares, embolismo pulmonar y miocardiopatía dilatada en una paciente con síndrome antifosfolípido. Arch Cardiol Mex. 2017;87(4):1-4.

8. Keeling D, Mackie I, Moore G, Greer I, Greaves M. Guidelines on the investigation and management of antiphospholipid syndrome. Br J Haematol. 2012;157(1):47-58.

9. Ford HJ, Roubey RA. Pulmonary manifestations of the antiphospholipid antibody syndrome. Clin Chest Med. 2010;31(3):537-45.

10. Ulrich S. Pulmonary Vasculitis and Hemorrhage. En: Spiro S, Silvestri G, Agustí A. Clinical Respiratory Medecine. 4th ed. Filadelfia: El Sevier; 2012. p. 722-30.

11. Isshiki T, Sugino K, Gocho K, Furuya K, Shimizu H, Sekiya M, et al. Primary Antiphospholipid Syndrome associated with diffuse alveolar hemorrhage and pulmonary thromboembolism. Intern Med. 2015;54(16):2029-33.

12. Kearon C, Akl EA, Ornelas J, Blaivas A, Jimenez D, Bounameaux $\mathrm{H}$, et al. Antithrombotic therapy for VTE disease. Chest. 2016;149(2):315-52.

13. Stojanovich L, Kontic M, Djokovic A, Ilijevski N, Stanisavljevic N Marisavljevic D. Pulmonary events in antiphospholipid syndrome: influence of antiphospholipid antibody type and levels. Scand J Rheumatol. 2012;41(3):223-6.

14. Danowski A, Rego J, Kakehasi A, Funke A, Carvalho JF, Lima IV, et al. Guidelines for the treatment of antiphospholipid syndrome. Rev Bras Reumatol. 2013;53(2):184-92.

15. Williamson JM, Dalton RS, Chester JF. Popliteal venous aneurysm causing pulmonary embolism and paradoxical embolisation in a Patient with antiphospholipid syndrome. Eur J Vasc Endovasc Surg. 2008;36(2):227-9.

16. Agnelli G, Becattini C. Acute Pulmonary Embolism. N Engl J Med. 2010;363(3):266-74.

17. Bĕlohlávek J, DytrychV, Linhart A. Pulmonary embolism, part I: Epidemiology, risk factors and risk stratification, pathophysiology, clinical presentation, diagnosis and nonthrombotic pulmonary embolism. Exp Clin Cardiol. 2013;18(2):129-38.

18. Kravitz MS, Shoenfeld Y. Trombocytopenic conditions-autoimmunity and hypercoagulability: commonalities and differences in ITP, TTP, HIT and APS. Am J Hematol. 2005;80(3):232-42.

19. Young-Joon Y, Gak-Won Y, Ik-Chan S, Seung-Woo B, KyuSeop L, Hye-Won R, et al. Clinical implications of elevated antiphospholipid antibodies in adult patients with Primary Immune Thrombocytopenia. Korean J Intern Med. 2011;26(4):449-54 